ÉGYPTE monde arabe

\section{Égypte/Monde arabe}

33 | 1998

Travailleurs et industrie à l'heure des réformes

économiques

\title{
Le mouvement ouvrier et la lutte contre la paupérisation
}

\section{Huwayda 'Adlî}

Traducteur : Samia Rizk

\section{(2) OpenEdition}

\section{Journals}

Édition électronique

URL : https://journals.openedition.org/ema/1580

DOI : 10.4000/ema. 1580

ISSN : 2090-7273

Éditeur

CEDEJ - Centre d'études et de documentation économiques juridiques et sociales

\section{Édition imprimée}

Date de publication : 30 juin 1998

Pagination : 113-124

ISSN : 1110-5097

\section{Référence électronique}

Huwayda 'Adlî, « Le mouvement ouvrier et la lutte contre la paupérisation », Égypte/Monde arabe [En ligne], 33 | 1998, mis en ligne le 08 juillet 2008, consulté le 07 juillet 2022. URL : http:// journals.openedition.org/ema/1580 ; DOI : https://doi.org/10.4000/ema.1580

Ce document a été généré automatiquement le 7 juillet 2022.

Tous droits réservés 


\title{
Le mouvement ouvrier et la lutte contre la paupérisation
}

\author{
Huwayda 'Adlî \\ Traduction : Samia Rizk
}

1 L'économie égyptienne a connu au cours de ces dernières années des changements structurels radicaux suite à la réorientation de sa politique économique. Dans le but de s'intégrer au marché capitaliste mondial, l'Égypte a en effet adopté les programmes d'ajustement structurel préconisés par les institutions internationales et engagés en conséquence des mesures de libéralisation de l'économie et de privatisation des entreprises publiques. La littérature portant sur l'intégration des économies des pays $\mathrm{du}$ Tiers-Monde dans le marché capitaliste mondial contient nombre d'accusations, les unes relatives aux répercussions sociales, les autres aux effets politiques de l'application des mesures de libéralisation économique, telles que l'augmentation du taux de chômage, l'accroissement de la pauvreté, la marginalisation sociale et l'instabilité politique croissante. Enfin, l'étude des conséquences de l'ajustement structurel sur la classe ouvrière dans les pays en développement montre une nette détérioration de leur situation, dont les premiers signes se sont manifestés dès les années soixante-dix avec l'aggravation de la crise économique dans ces pays. Celle-ci s'est traduite, au niveau extérieur, par la chute des revenus à l'exportation, la hausse des prix à l'importation, l'augmentation du déficit de la balance des paiements, ainsi que l'augmentation des taux d'inflation et du poids de la dette extérieure. Au niveau intérieur, elle s'est traduite par l'aggravation du déficit du budget de l'état, l'augmentation des taux d'inflation, la régression des taux de l'épargne et des investissements et par la corruption qui a permis à des catégories parasites d'exploiter la crise et d'amasser d'immenses fortunes grâce à la spéculation, aux pots-de-vin, à la fraude ou la contrebande. Les répercussions des politiques et des programmes d'ajustement structurel sur la classe ouvrière des pays en développement sont à recenser et à analyser à deux niveaux : la distribution du revenu intérieur et le niveau de vie de la classe ouvrière. 
2 Concernant le premier niveau, l'expérience montre que la part des salaires dans le produit intérieur brut se réduit à l'avantage des propriétaires des facteurs de production, c'est-à-dire de ceux qui perçoivent profits, intérêts et rentes. Selon des études récentes visant à connaître les effets de ces programmes d'ajustement structurel (PAS) sur un certain nombre de pays d'Amérique latine et des Caraïbes durant la période 1980-1985, période durant laquelle ils ont appliqué les PAS, le secteur des industries manufacturières et celui du bâtiment ont connu, respectivement, une réduction de $8,4 \%$ et de $19,4 \%$ des salaires réels des travailleurs, tandis que le salaire minimum baissait de 11,8\%. Par ailleurs, la part de la population qui vit au-dessous du seuil de pauvreté absolue est passée de $21 \%$ à $29 \%$ dans les villes, et elle a augmenté de $54 \%$ en milieu rural ${ }^{1}$.

3 À un deuxième niveau - celui du niveau de vie de la classe ouvrière -, le caractère déflationniste des PAS, conçus de manière à réduire la demande de biens de consommation et d'investissement, a pour effet d'augmenter le chômage. Celui-ci constitue un véritable danger dans les pays à forte croissance démographique, où le nombre de personnes arrivant chaque année sur le marché du travail est important. Rappelons qu'il existe deux sortes de chômeurs : ceux qui ont perdu leur emploi et ceux qui arrivent sur le marché du travail pour la première fois. On sait que le poids du chômage dans les pays en développement est plus pénible et plus douloureux que dans les pays industriels capitalistes, où existe un système d'indemnisation et de protection sociale qui assure aux travailleurs un revenu minimum.

Une autre conséquence des programmes de privatisation qui pèse sur les travailleurs est la forte réduction de leurs salaires réels du fait de la hausse des prix ; celle-ci est aggravée par la suppression des subventions sur les produits du secteur public, de l'introduction des mécanismes de l'offre et de la demande sur tous les marchés, ainsi que de la compression des dépenses publiques dans le domaine des services sociaux indispensables tels que l'éducation et la santé.

5 Par ailleurs, le transfert de propriété des entreprises publiques vers les secteurs privé et étranger a entraîné la mise à pied d'un grand nombre de salariés et une réduction des salaires, des avantages et des droits acquis. Ce n'est pas un hasard si, avec les progrès de la privatisation, les hommes d'affaires réclament de plus en plus fort un amendement du code du travail afin de réduire les coûts de production aux dépens du facteur humain, ce qui pousse les salariés soit à s'adapter, soit à résister.

6 Dans le présent document nous nous intéresserons en premier lieu à l'étude des mécanismes de résistance contre la paupérisation et la marginalisation au sein du mouvement ouvrier. Par résistance, nous entendons les différentes formes d'opposition, directe ou indirecte, aux politiques d'ajustement structurel par la voie de la contestation collective: grèves, occupations d'usines, manifestations, refus de percevoir les salaires en signe de protestation contre les nouvelles conditions de travail ou de vie découlant de l'adoption des politiques économiques de libéralisation.

7 Notons qu'il existe une différence radicale entre les mécanismes de résistance et les mécanismes d'adaptation. Dans ce dernier cas, c'est en désespoir de cause que le salarié cherche à s'adapter à la nouvelle situation, individuellement ou collectivement.

8 La présente étude couvre les années 1987 à 1993, période d'accélération des opérations de privatisation : c'est-à-dire, de la signature de la lettre d'intention par l'État égyptien, aux premières mesures effectives de privatisation des entreprises publiques, en passant 
par diverses décisions politiques préparant le terrain à la promulgation de la loi 203 de 1991 relative au nouveau "secteur des affaires». Elle s'appuie sur deux types de sources : les rapports stratégiques annuels que publie le Centre d'études politiques et stratégiques d'al-Ahrâm et les recherches publiées par le mouvement ouvrier; et la presse nationale gouvernementale et d'opposition, après vérification des données publiées.

9 Toutefois, il ne faut pas perdre de vue au cours de ce travail de vérification que la publication des données relatives à la violence politique en général, et aux protestations ouvrières en particulier, fait l'objet d'importantes réserves. On distingue deux attitudes : la première cherche à minimiser au plus haut degré la portée de ces mouvements et leur importance; la second tend au contraire à dramatiser le phénomène. Le lecteur est sûrement conscient du parti pris que comporte chacune de ces attitudes.

10 Cette étude se propose de répondre aux questions suivantes :

$111^{\circ}$ ) Quelles sont les causes et les revendications du mouvement de contestation de la classe ouvrière en Égypte?

$122^{\circ}$ ) Quels sont les secteurs ouvriers où se concentrent les mécanismes de résistance et de contestation?

$133^{\circ}$ ) Comment évolue l'action contestataire et quels en sont les résultats?

$\left.144^{\circ}\right)$ Comment l'État affronte-t-il de tels mouvements ? Les modalités de la confrontation ont-elles évolué ?

$\left.155^{\circ}\right)$ Qu'en est-il des relations entre les mouvements contestataires des ouvriers et l'organisation syndicale?

16 Nous procéderons ici à une analyse qualitative de ces mouvements pour rendre compte du phénomène dans son évolution et sa dynamique, et non des seuls faits. Pour ce faire, nous exposerons le phénomène depuis son apparition, les phases de son évolution, les résultats auxquels il a abouti et la position de toutes les parties concernées.

17 Cela ne signifie cependant pas que nous ignorerons l'aspect quantitatif du phénomène ; celui-ci sera implicitement traité dans notre étude.

18 Par ailleurs, pour une claire interprétation des phénomènes sociaux et politiques, nous utiliserons la méthode comparative qui permet de montrer les spécificités, les différences et les ressemblances entre les différents moments de l'évolution du phénomène et, par conséquent, de dresser la courbe correspondante. Par évolution, nous entendons ici, non pas uniquement la croissance, mais aussi la régression, c'est-àdire ses flux et ses reflux.

19 Nous étudierons donc deux périodes successives : 1982-1987 et 1987-1993.

\section{L'évolution du phénomène contestataire au sein du mouvement ouvrier entre 1982 et 1987}

20 D'après ${ }^{2}$ les données disponibles, la période de 1982-1984 est relativement calme. Aucune contestation ouvrière n'a été relevée ni dans la presse officielle, ni dans la presse de l'opposition, ni dans le rapport stratégique annuel d'al-Ahrâm. Ce calme peut 
s'expliquer par les changements survenus au niveau politique et par l'expectative des travailleurs, incertains de leur sort sous la nouvelle direction.

La période 1984-1987 connait une progression lente du phénomène contestataire. Alors que durant les années 1984-1985 il n'y a eu que trois grèves (dans l'usine de textile de Kafr al-Dawwâr en septembre 1984 ; dans la Société d'industries électromécaniques de Shubra (usine militaire 27) en novembre 1984, et dans l'usine militaire 18 d'Abû Za'bal en décembre 1985), l'année 1986 a connu 13 grèves ouvrières ${ }^{3}$, dont 9 dans le secteur industriel et 4 dans le secteur tertiaire.

Ce qui préoccupe le plus les ouvriers et les amène à se mettre en grève, ce sont les problèmes catégoriels (notamment les salaires, les primes d'encouragement, les conditions de travail et les prix). Les revendications ont un caractère partiel en ce sens qu'elles sont limitées aux problèmes que nous venons de citer et à l'unité de production elle-même.

Les ouvriers ne recourent à la grève qu'après avoir épuisé toutes les ressources et les moyens pacifiques pour faire entendre leurs revendications. Ils commencent par porter plainte, puis font intervenir le syndicat de branche, demandent à rencontrer les directeurs et enfin seulement menacent de faire grève.

À de rares exceptions près, la plupart des actions de contestation ont été menées sans l'approbation des syndicats et contre leur volonté. Le rôle de l'organisation syndicale s'est limité... à dénoncer les grèves ou, dans le meilleur des cas, à jouer le rôle d'intermédiaire entre la direction et les travailleurs, le plus souvent après une forte détérioration de la situation. Telles sont, en bref, les caractéristiques du phénomène contestataire durant les années 1982-1987.

\section{La contestation ouvrière entre 1987 et 1993}

Cette période connaît une accélération du mouvement contestataire ouvrier. En effet, entre les grèves, les manifestations, les occupations d'usines, le refus de percevoir les salaires et les grèves de la faim, on assiste à 111 actions contestataires, soit une moyenne de 15 par an.

Concernant la répartition de ces actions sur les différents secteurs ouvriers, 80 sur 111, soit $72 \%$, sont le fait du secteur industriel, le reste étant réparti entre le secteur tertiaire, en particulier les transports et les communications, et le secteur agricole. Dans le secteur industriel, les branches de la métallurgie, des industries électromécaniques et de l'industrie chimique sont les plus touchées par ce phénomène. La concentration de la contestation dans le secteur industriel en général, et plus particulièrement, dans les industries susmentionnées, est liée à un degré de conscience collective plus élevé dans le milieu industriel que dans ceux des services ou des activités agricoles, en raison de leur regroupement dans un lieu unique et de la pratique d'un type de production supérieur.

28 Au cours de ces années, le phénomène a évolué de la manière suivante : en 1987, 4 actions ; en 1988, 16 ; en 1989, 21 actions ; en 1990, 12 ; en 1991, 9 actions ; en 1992, 24 ; et en 1993, 25 actions.

29 Notons l'amplification du phénomène d'année en année et le sommet atteint en 1992-1993. Quant à la relative régression de 1991, elle s'explique par le fait que les 
ouvriers étaient occupés à la préparation des élections syndicales. En revanche, la progression est liée à la dégradation de leurs conditions de vie résultant de l'accélération de l'application des politiques d'ajustement structurel et de la hausse des prix des biens et des services qui s'en est suivie, d'où une baisse des salaires réels; d'autre part, les opérations de liquidation des entreprises publiques et la vente de ces dernières ont été entamées avec la promulgation de la loi 203 de 1991. En conséquence, l'État s'est désengagé financièrement de la subvention du secteur public, ce qui s'est traduit par la mise à pied d'un grand nombre de travailleurs, notamment ceux sous contrats à durée déterminée ou annuels, par des retards de plusieurs mois dans le versement des salaires, par une baisse du salaire variable (primes et indemnités) alors que ce dernier représente une part importante du salaire total (et atteint entre $150 \%$ et $300 \% \mathrm{du}$ salaire de base). C'est ce que l'analyse du mouvement revendicatif des ouvriers égyptiens permettra de constater de manière évidente.

Notons que la crise économique et la menace du chômage ne touchent pas uniquement les travailleurs du secteur public, mais aussi ceux du secteur privé, surtout dans le domaine des industries nationales qui se trouvent confrontées à de graves problèmes depuis la libéralisation des importations. Cela a conduit à la liquidation de plusieurs industries et à la mise à pied d'une grande partie de la main-d'œuvre.

31 L'analyse des revendications ouvrières permet de constater que la situation n'est pas différente de celle qu'a connue la période précédente (1982-1987). Les problèmes catégoriels - niveau des salaires, réduction des primes, non-versement des allocations et des bonus - sans compter les mauvaises conditions de travail, sont toujours à l'ordre du jour et suscitent le plus de contestations.

Nous pouvons donc dire que ces mouvements contestataires ne sont en fait rien d'autre que des tentatives de la part des ouvriers de s'opposer à la politique étatique de réduction du coût de la production aux dépens des travailleurs. À cela s'ajoute, durant cette période 1987-1994, la protestation contre les licenciements des travailleurs temporaires et le refus de renouveler leurs contrats. Ces mouvements dénoncent aussi la mauvaise gestion des unités de production, qui s'est traduite par l'entassement et la détérioration des marchandises dans les entrepôts, par l'interruption du travail sur de nombreuses lignes de production et par l'absence d'entretien du matériel de production. L'intensité du conflit opposant la direction aux salariés s'est manifestée dans plusieurs mouvements où les ouvriers ont imputé à la direction la responsabilité des pertes de l'entreprise et l'ont accusée de corruption et d'abus de pouvoir. De même, la fusion des entreprises en vertu de la loi 203 de 1991 a suscité de vives contestations.

Nous remarquons donc un changement dans la nature des revendications elles-mêmes. Les ouvriers sont maintenant clairement conscients du lien qui existe entre les nouvelles politiques économiques adoptées et la détérioration de leurs conditions de vie, ainsi que le risque encouru, non seulement de voir leur salaire baisser, mais aussi de perdre leur emploi

34 Les incidents de Kafr al-Dawwâr, qui ont débuté le 30 septembre 1994, confirment en quelque sorte cette prise de conscience. L'analyse du mouvement montre qu'il visait essentiellement la politique de privatisation. La politique adoptée par la direction de l'entreprise a consisté à utiliser des mesures vexatoires envers les travailleurs pour se débarrasser d'un grand nombre d'entre eux ou pour réduire la partie variable des salaires. Ce mouvement de protestation a été provoqué par le licenciement de 2000 ouvriers sous contrats temporaires depuis plusieurs années et le transfert de 70 autres 
dans les filiales de Mahmûdiyya et de Kûm Hamâda, sans compter les sanctions abusives qui leur étaient infligées. L'Agence centrale des comptes a elle-même critiqué la rigueur de ces sanctions.

Toutes ces mesures ne visaient que la mise en place de l'ajustement structurel en cherchant à réduire les coûts de production aux dépens du facteur humain. Dans un entretien accordé par M. 'Âtif 'Ubayd, ministre du Secteur public des Affaires, à la revue Al-Musawwar du14 octobre 1994, à la suite des incidents de Kafr al-Dawwâr, il apparaît que la réduction des salaires, tout comme l'amélioration de la production et le recouvrement des créances, est considérée comme l'un des mécanismes permettant de réduire les coûts et de redresser les entreprises. Au cours de cet entretien, le ministre répète que, dans ses rencontres avec les présidents des holdings, il a insisté pour que la réduction des salaires ne soit pas le seul moyen envisagé et que d'autres mesures soient examinées. Il n'en reste pas moins que, dans la pratique, l'État laisse l'entière liberté à la direction des entreprises pour décider des moyens d'accélérer la liquidation des sociétés.

D'autre part, de par son ampleur, tant sur le plan de la durée que du nombre de participants et de revendications, le mouvement de Kafr al-Dawwâr a eu un fort retentissement dans d'autres usines telles que les usines textiles de Hilwân et de Mahalla. Dans la même semaine, pressentant que la direction de l'usine avait l'intention de réduire le coût de la production à leurs dépens, les ouvriers de Hilwân présentent des revendications relatives aux primes d'encouragement et à l'indemnité de repas. Peu après ces incidents, surviennent ceux de l'usine textile de Mahalla : un groupe de leaders ouvriers s'est réuni et a décidé d'organiser une marche pour demander au comité syndical de transmettre à la direction leurs revendications concernant essentiellement l'augmentation de la prime d'encouragement et de l'indemnité de repas, ainsi que la destitution du président du conseil d'administration.

En réaction à ces mouvements (nous ne parlons pas ici des réactions relatives à la sécurité), MM. 'Âtif 'Ubayd, Ahmad al-'Ammâwî et al-Sayyid Râshid ${ }^{4}$ se sont rendus sur différents lieux de production à Hilwân, Tanta, Alexandrie et Asyût, pour voir quels étaient les problèmes réels des ouvriers. Au cours de ces rencontres, des confrontations ont eu lieu entre les représentants des syndicats et les responsables au sujet des méfaits de la politique de privatisation et des modalités de sa mise en œuvre. Pour montrer que les mouvements contestataires représentaient un changement qualitatif ayant des répercussions sur d'autres entreprises industrielles et de service, il suffit de citer les incidents de l'usine sidérurgique de Hilwân en 1989, où la principale revendication portait sur l'augmentation de l'indemnité de repas. À la suite de ce mouvement, les responsables furent contraints de verser une indemnité de repas dans plusieurs autres usines, telles que les usines de laminage, de coke et de tuyauteries à Hilwân, sans qu'il n'y ait eu de mouvements contestataires dans ces entreprises.

La même situation se reproduit, quoique de manière un peu différente, en septembre 1988, lorsque les ouvriers de l'usine textile de Mahalla al-Kubra font grève pour protester contre la suppression de l'allocation versée à l'occasion de la rentrée scolaire et contre la hausse des prix de certains produits. L'allocation est versée, mais sous une autre appellation. Des mouvements similaires se produisent en d'autres lieux (les usines de sucre et d'aluminium de Naga' Hamâdî, les chemins de fer à Suhâg, l'usine d'apprêtage des tissus à Mahalla al-Kubra et l'usine militaire 999) et les ouvriers réussissent à obtenir le versement de l'allocation, toujours sous une autre appellation. 
39 Il n'est nul besoin de démontrer qu'un mouvement contestataire de cette envergure, qui se produit dans des régions telles que Mahalla al-Kubra ou Hilwân ou Kafr alDawwâr et dans une usine qui compte plus de dix mille salariés a une incidence tout autre que celle d'un petit établissement où la concentration ouvrière est nettement plus faible.

40 Le mouvement ne comportait que d'anciennes revendications jamais satisfaites par la direction. Eu égard à l'évolution de l'action revendicative, les ouvriers n'avaient recours à la contestation collective, que ce soit sous forme de grève, d'occupation d'usine ou autres, que lorsqu'ils avaient épuisé tous les autres moyens pacifiques leur permettant d'obtenir satisfaction : plaintes portées devant le conseil d'administration, intercession du comité syndical ou du syndicat de branche, menaces de grèves dans des dépêches adressées au président de la République, au Premier ministre, au ministre de l'Industrie, au ministre du Secteur public des affaires et à d'autres responsables. Et ce n'est que lorsque toutes ces tentatives se sont soldées par un échec que les ouvriers ont eu recours aux grèves et aux manifestations.

41 Par ailleurs, concernant l'organisation syndicale, on note un changement de position par rapport aux mouvements contestataires. Contrairement à ce qui s'est produit dans le passé, celle-ci ne dénonce plus les contestations ouvrières et ne les désavoue plus. Ce changement s'est fait sentir à partir de 1990. Dans tout ce qui a été rapporté sur la position de l'organisation syndicale, on constate qu'elle ne cherche plus à se tenir à l'écart comme par le passé et qu'elle prend une part active dans l'action qui est menée en tenant des réunions avec la direction ou en soumettant une note au ministre compétent, voire en attribuant le déficit de l'entreprise à la mauvaise gestion et aux querelles de l'administration. Dans certains cas (rares il est vrai), il est même arrivé que le comité syndical en arrive à présenter sa démission, en signe de protestation face à une direction qui refusait de satisfaire les revendications des ouvriers, ou participe au mouvement contestataire de manière effective.

42 C'est ainsi qu'en juin 1990, devant le refus de satisfaire les revendications des ouvriers de l'usine de tapisserie de Mahalla, le comité syndical de l'entreprise a présenté une démission collective. En juin 1993, le comité syndical de la Société de tabac de Tanta a participé aux manifestations organisées par les ouvriers de l'entreprise.

43 La grève des ouvriers des mines et carrières de juin 1994 est un exemple de ce changement qualitatif. Dans un geste sans précédent dans l'histoire de l'organisation syndicale officielle depuis 1957, le conseil d'administration du syndicat des ouvriers des mines et carrières lance un mot d'ordre de grève générale en application des décisions de l'assemblée générale du syndicat réunie le 29 mai 1994. Cet événement se distingue par le fait qu'il s'agit d'une action authentique et non d'une réaction comme cela se produit lorsqu'une représentation syndicale, de quelque niveau que ce soit, prend part à une action contestataire. De plus, cela s'est produit à un niveau plus élevé que d'habitude, celui du syndicat de branche, et non à celui des comités comme auparavant.

Ce changement indique que l'organisation syndicale est à présent consciente qu'il est vain de continuer à entretenir un lien étroit avec les autorités politiques, d'autant qu'avec le démantèlement du secteur public, ce lien n'a plus de raison d'être. Pour continuer à exister, les syndicats doivent assumer le rôle qui leur incombe normalement, à savoir la défense des droits des travailleurs. 
ela ne veut pas dire que le syndicalisme au service du pouvoir et hostile aux ouvriers a complètement disparu, ou que les syndicats et les ouvriers partagent la même position et les mêmes intérêts. Des divergences radicales entre les deux parties persistent. Certes, l'organisation syndicale accorde un appui plus franc et plus courageux aux revendications des ouvriers, mais elle estime toutefois que la solution réside dans les négociations avec la direction, même si ces dernières durent indéfiniment et ne prennent fin qu'avec l'extinction même des revendications.

L'évolution du phénomène contestaire apparaît également dans les faits suivants :

1. un nouveau phénomène se manifeste dans les relations des organes de sécurité avec les mouvements contestataires. Ceux-ci ne se contentent plus de contenir le mouvement, mais ils jouent aussi le rôle d'intermédiaire entre les ouvriers et la direction, ce qui se justifie peut-être par le souci de maintenir un minimum de stabilité politique.

2. Les formes de contestation ne se limitent plus aux grèves, aux manifestations ou autres formes susmentionnées. Un nouveau mécanisme est mis en place : il s'agit du recours à la justice pour arrêter la vente ou la liquidation d'un établissement, ou encore pour dénoncer le caractère anticonstitutionnel de la loi sur le secteur des affaires.

3. On assiste à nouveau à des grèves communes, c'est-à-dire à des grèves organisées en même temps dans différentes usines ou différents établissements.

De manière générale et dans les limites des données disponibles ${ }^{5}$, on constate que les résultats de l'action contestataire varient entre la satisfaction ou la réalisation partielle des revendications, et la promesse de les étudier. En fait, l'efficacité de ces luttes dépend d'un certain nombre de facteurs comme: $1^{\circ}$ ) le type d'industrie, sensible ou stratégique $; 2^{\circ}$ ) le lieu géographique : que la contestation se produise dans une région à forte concentration ouvrière ou connue pour son passé combatif, comme la région de Mahalla al-Kubra et Kafr al-Dawwâr, ou dans un petit établissement dans une région à faible densité ouvrière, l'impact n'est pas le même $; 3^{\circ}$ ) le nombre de participants ; $4^{\circ}$ ) la durée de la grève $; 5^{\circ}$ ) le niveau d'organisation et $6^{\circ}$ ) la position du syndicat par rapport à cette grève.

De même que certaines caractéristiques (la sensibilité, l'importance, l'emplacement géographique, l'accumulation de problèmes et le degré d'insatisfaction) contribuent à amplifier l'impact et l'effet du mouvement contestataire, d'autres facteurs en limitent l'efficacité : les uns sont liés à l'absence des caractéristiques que nous venons de citer, les autres relèvent de la situation économique dans son ensemble, c'est-à-dire du taux de chômage. En effet, un taux de chômage élevé et par conséquent l'existence d'une «armée» de chômeurs ou d'emplois précaires limitent dans une large mesure l'efficacité de la contestation.

\section{NOTES}

1. Ramzî ZAKÎ, 1993, al-lîbirâliyya al-mustabidda (Le libéralisme despotique), Le Caire, Sîna li-lnashr, p. 118-120.

Égypte/Monde arabe, 33 | 1998 
2. Cette partie s'appuie sur une étude présentée par l'auteur au colloque «L'étude de la société égyptienne et les préoccupations des jeunes chercheurs" sous le titre "Le mouvement contestataire de la classe ouvrière égyptienne 1982-1991» et publiée dans Préoccupations de l'Égypte et crise des jeunes cerveaux, Le Caire, 1994.

3. La société ESCO de Shubra al-Khayma, la société Misr pour la filature et le tissage, la société Arab Investments pour les industries électriques au 10-Ramadan, l'hôtel Saphir à Zamalek, les conducteurs de chemins de fer, la société ETCO pour l'industrie du bois, les mêmes ouvriers de ETCO, le garage al-Munîb, les ouvriers du métro, la société de filature et de tissage de Mahalla alKubra, l'usine de batteries, les ouvriers des ateliers d'irrigation, l'usine de tapisserie de la coopérative des industries de l'électro-ménager de Mahalla al-Kubra.

4. Ahmad al-'Ammâwî est ministre de la Main-d'œuvre et al-Sayyid Râshid président de la Fédération syndicale (N.D.E.).

5. Une ébauche de tableau chronologique recensant les mouvements de contestation ouvrière entre 1987 et 1993 figurait dans la communication en arabe dont est tiré cet article. Cette dernière fut présentée dans le cadre du colloque « Les effets de la politique de privatisation sur la situation des travailleurs et des pauvres des régions urbaines ", qui s'est tenu à Hilwân, du 22 au 28 février 1997, au Centre d'aide syndicale et ouvrière (Dâr al-khadamât al-niqâbiyya wal-'ummâliyya). L'absence de données statistiques n'a pas permis à l'auteur de compléter les informations par trop lacunaires, puisées dans la seule presse égyptienne.

\section{INDEX}

Mots-clés : mouvement ouvrier, paupérisation, pauvreté

\section{AUTEURS}

\section{HUWAYDA 'ADLî}

CNRSC (Centre national de recherches sociologiques et criminologiques), Le Caire 